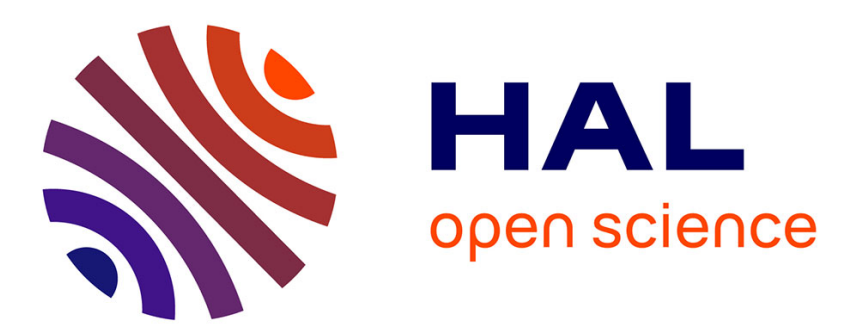

\title{
Quasistatic rheology and microstructural description of sheared granular materials composed of platy particles
}

\author{
Mauricio Boton, Emilien Azéma, Nicolas Estrada, Farhang Radjai, Arcesio
}

Lizcano

\section{- To cite this version:}

Mauricio Boton, Emilien Azéma, Nicolas Estrada, Farhang Radjai, Arcesio Lizcano. Quasistatic rheology and microstructural description of sheared granular materials composed of platy particles. Physical Review E : Statistical, Nonlinear, and Soft Matter Physics, 2013, 87 (032206), pp.1-15. 10.1103/PhysRevE.87.032206 . hal-00759623

\section{HAL Id: hal-00759623 \\ https://hal.science/hal-00759623}

Submitted on 28 Mar 2013

HAL is a multi-disciplinary open access archive for the deposit and dissemination of scientific research documents, whether they are published or not. The documents may come from teaching and research institutions in France or abroad, or from public or private research centers.
L'archive ouverte pluridisciplinaire HAL, est destinée au dépôt et à la diffusion de documents scientifiques de niveau recherche, publiés ou non, émanant des établissements d'enseignement et de recherche français ou étrangers, des laboratoires publics ou privés. 


\title{
Quasistatic rheology and microstructural description of sheared granular materials composed of platy particles
}

\author{
Mauricio Boton, ${ }^{1,2, *}$ Emilien Azéma, ${ }^{2, \dagger}$ Nicolas Estrada, ${ }^{1}$ Farhang Radjaï, ${ }^{2}$ and Arcesio Lizcano ${ }^{1}$ \\ ${ }^{1}$ Departamento de Ingeniería Civil y Ambiental, CeiBA Complex Systems Research Center, Universidad de Los Andes, Bogotá, Colombia \\ ${ }^{2}$ LMGC, Université Montpellier 2, CNRS, Place Eugène Bataillon, 34095 Montpellier cedex 05, France \\ (Received 28 November 2012; revised manuscript received 14 February 2013; published 28 March 2013)
}

\begin{abstract}
This is the first paper of a series devoted to the micro-mechanical modeling of clayey soils, by means of discrete element simulations. We specifically focus here on the effect of the platy shape of particles by reducing the interactions between particles to mechanical contact forces (i.e., neither electrostatic repulsion nor van der Waals forces are taken into account). The particles are three-dimensional square plates, approximated as spheropolyhedra. Several samples composed of particles of different levels of platyness (related to the ratio of length to thickness) were numerically prepared and sheared up to large deformations. We analyzed the shear strength, packing fraction, orientation of the particles, connectivity, fabric of the interactions network, and interaction forces as functions of the platyness. We find that both the mechanical behavior and microstructure are strongly dependent on the degree of platyness. The principal underlying phenomenon is the alignment of particle faces along a particular direction. This ordering phenomenon, which emerges even for shapes that deviate only slightly from that of a sphere, enhances the ability of the packing to develop an anisotropic structure leading to large shear strength, especially as a consequence of the fabric and mobilization of friction forces. Moreover, the connectivity of the packings and their packing fraction also evolve with the platyness. In particular, the packing fraction evolves in a nonmonotonic fashion, as observed in other granular materials composed of elongated or angular particles.
\end{abstract}

DOI: 10.1103/PhysRevE.87.032206

PACS number(s): 45.70.-n, 83.80.Fg, 61.43.-j

\section{INTRODUCTION}

Over the past two decades, simulations with discrete element methods (DEM) have proven to be a privileged analysis tool in various domains of science and engineering. One of these domains is soil mechanics [1], for which DEM simulations are useful because they allow for exploring, based on the scale of the grains and their interactions (the microscale), various phenomena that have been investigated experimentally for decades, or even centuries for some properties. As surprising as this may seem, many of these phenomena, which are considered today to be quite classical for the mechanical description of the material at the scale of an elementary representative volume (the macroscale), still lack a clear, physically based explanation. Examples of such phenomena are internal friction, strain localization, strain-rate dependent behavior, creep, and stress relaxation [2]. Obviously, the technical difficulties associated to the experimental observation of what really happens at the microscale are some of the reasons why the mechanisms at the origin of these phenomena have remained elusive.

However, the application of DEM for the investigation of soils has been restricted to a class of soils: coarse soils. Soils not belonging to this class (i.e., fine soils, such as clays) have been left out of the scope of this analysis tool. The reasons why DEM simulations of fine soils are difficult, when compared to those of coarse soils, can be understood when analyzing two essential differences between the two materials. The first difference is the shape of the particles. On one hand, particles in a coarse soil typically have bulky shapes. These particles can

\footnotetext{
*cm.boton131@uniandes.edu.co

†emilien.azema@univ-montp2.fr
}

be approximated by simple shapes such as disks or spheres, which are easy to deal with in a numerical simulation. On the other hand, particles in a fine soil typically have platy shapes. These shapes are difficult to manage efficiently in a numerical simulation, because of tasks such as contact detection. The second difference is the interaction between particles. On one hand, the interactions between coarse particles can be reduced to contact forces, usually decomposed in a normal repulsion force and a friction force (additionally, a cohesion force is introduced in the case of partially saturated or cemented materials). These interactions can be prescribed as simple contact laws such as the linear spring-dashpot model and Coulomb law. On the other hand, the interactions between fine particles include, besides contact forces, electrostatic repulsion due to chemical interactions and fluid, and attraction due to van der Waals forces. These interactions cannot be prescribed as contact laws, but require an integration procedure over the particle surfaces, which can be extremely time consuming in simulations with a large number of particles. These technical problems explain why DEM simulations of fine soils are so scarce [3-6].

This paper is the first of an ongoing investigation devoted to the mechanical behavior of clayey soils by means of DEM simulations. Specifically, the aim of the present work is to explore the effect of the platy shape of particles, typically observed in clay particles, on the mechanical behavior and microstructure of a granular material. Singling out the effects of particle shape as compared to other characteristics such as the complex particle-particle or particle-fluid interactions is an important step forward in a general modeling strategy of clays. For this reason, in this paper we consider only "dry" assemblies of particles, and these particles interact only through "conventional" interactions, i.e., normal repulsion and 
friction forces, as traditionally implemented for simulating coarse granular materials. In this sense, the findings presented in this paper are rather generic and apply also to other granular materials made up of flat particles. A few examples could be granular soils derived from shale, edible seeds such as lentils, and industrial goods such as PET flakes.

The simulated particles are square plates, which we approximate as spheropolyhedra [7-9] simulated by means of the soft-sphere Molecular Dynamics (MD) method. The degree of platyness is described by a parameter $\eta$, related to the ratio of length to thickness, and is varied systematically from $\eta=0$, which corresponds to spherical particles, to $\eta=0.8$, which corresponds to particles 5 times longer than thick. Several monodisperse assemblies were built numerically, one for each value of $\eta$, and then sheared in the quasistatic limit up to the "critical state" (as termed in the soil mechanics literature), where the memory of the initial state is fully erased and a steady shear state is reached. In this state we analyze the mechanical behavior of the material at the macroscale, in terms of shear strength and packing fraction, as well as its microstructure, in terms of particle connectivity and orientations, fabric of the interaction network, and forces.

In the following, we introduce the numerical model, system characteristics, and simulation parameters in Sec. II. In Sec. III we focus on the mechanical behavior of the material at the macroscale, and, in Sec. IV, we analyze its microstructure. Finally, in Sec. V we end with a summary of the most salient results followed by a brief discussion.

\section{NUMERICAL MODEL}

\section{A. Platy shaped particles}

The particles are square plates with rounded edges, built as a sphero-polyhedra resulting from sweeping a sphere around a polyhedron. Mathematically, this corresponds to a Minkowsky addition of a sphere with a polyhedron (for some examples of nonspherical particles built as spheropolyhedra, see Refs. [11] and [7] for cylinders; Ref. [12] for particles with shapes as complex as that of a cow; Ref. [8] for cylinders, tetrahedra, and intersecting cylinders; and Ref. [13] for irregular polyhedra). Specifically, our particles are spheroplates resulting from a Minlowsky addition of a square plate and a sphere. Each of these plates has three kind of constitutive entities: (1) four vertices, (2) four edges, and (3) one plane, as shown in Fig. 1(a).
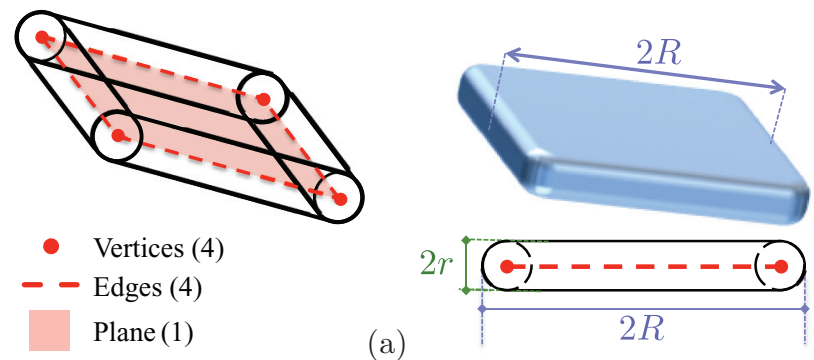

(b)

FIG. 1. (Color online) (a) Scheme of a spheroplate and its constitutive elements. (b) Definition of the maximum and minimum radii, $R$ and $r$, respectively.
The platyness $\eta$ of these spheroplates is defined as

$$
\eta=\frac{R-r}{R},
$$

where $R$ and $r$ are, respectively, the maximum and minimum radii of the spheroplate as defined in Fig. 1(b) $(r$ is also called the spheroradius of the spheroplate). Note that $\eta$ varies from 0 for a sphere to 1 for an infinitely thin plate. The platyness $\eta$ is related to the more common aspect ratio $\lambda$ through the simple expression $\eta=1-1 / \lambda$.

\section{B. Simulation method}

We employed the MD method, adapted first by Cundall and Strack for simulating granular materials [10]. In applying MD to spheroplates, we should distinguish the contacts between different elements (vertices, edges, and faces) of two interacting particles. Each interaction represents single or multiple contacts $c$, each contact occurring between two elements belonging to either of the spheroplates. All possible contacts are resolved by considering two cases: a contact between two edges and a contact between a vertex and a face [14]; see Fig. 2.

The forces $f$ at a contact between two elements are calculated using a linear spring-dashpot model. The normal component $f_{n}$ is given by

$$
\boldsymbol{f}_{n}=-\boldsymbol{n}\left\{\begin{array}{cc}
0, & \delta_{n} \leqslant 0, \\
k_{n} \delta_{n}+\gamma_{n} \dot{\delta}_{n}, & \delta_{n}>0,
\end{array}\right.
$$

where $\boldsymbol{n}$ is a unitary vector normal to the contact, $\delta_{n}$ is the normal overlap, $k_{n}$ is the normal stiffness, and $\gamma_{n}$ is the normal damping coefficient. The normal direction is defined as the direction of the vector $\boldsymbol{d}$ joining the two closest points belonging to the contacting elements (see Fig. 3). Note that this definition of the normal direction implies that all the

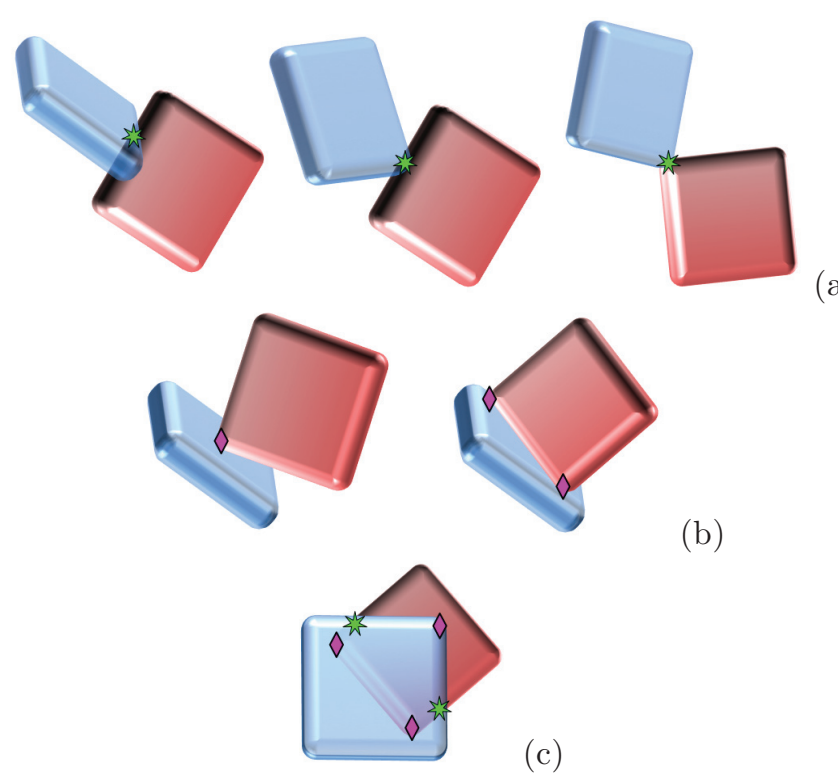

(a)

FIG. 2. (Color online) (a) Interactions comprising only edge-edge contacts (stars). (b) Interactions comprising only vertex-face contacts (diamonds). (c) Interactions comprising both edge-edge and vertexface contacts. 

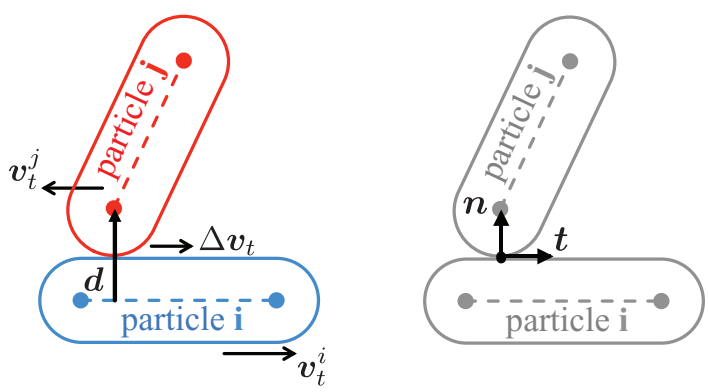

FIG. 3. (Color online) Definition of the vectors $\boldsymbol{d}$ and $\Delta \boldsymbol{v}_{t}$ used to define the normal $\boldsymbol{n}$ and tangential $\boldsymbol{t}$ directions of a contact between two elements.

contacts $c$ composing an interaction share the same vector $\boldsymbol{n}$. The normal overlap $\delta_{n}$ is given by $\left(r^{i}+r^{j}\right)-d$, where $r^{i}$ and $r^{j}$ correspond, respectively, to the spheroplates $i$ and $j$, and $d$ is the norm of $\boldsymbol{d}$. Note that the linear-spring dashpot model can lead to attractive forces when the particles are separating and $\delta_{n}$ approaches zero [15]. In our simulations, $f_{n}$ is set to zero whenever it becomes attractive. The tangential component $\boldsymbol{f}_{t}$ is given by

$$
\boldsymbol{f}_{t}=\boldsymbol{t} \begin{cases}\operatorname{sgn}\left(\hat{f}_{t}\right) \mu f_{n}, & \left|\hat{f}_{t}\right| \geqslant \mu f_{n}, \\ \hat{f}_{t}, & \left|\hat{f}_{t}\right|<\mu f_{n},\end{cases}
$$

where $t$ is a unitary vector tangent to the contact, $\mu$ is the friction coefficient, and $\hat{f}_{t}$ is a test function defined as

$$
\hat{f}_{t}=-k_{t} \delta_{t}-\gamma_{t} \dot{\delta}_{t}
$$

where $k_{t}$ is the tangential stiffness, $\delta_{t}$ is the tangential overlap, and $\gamma_{t}$ is the tangential damping coefficient. The tangential direction is defined as the direction of the vector $\Delta \boldsymbol{v}_{t}=\boldsymbol{v}_{t}^{j}-$ $\boldsymbol{v}_{t}^{i}$, where $\boldsymbol{v}_{t}^{i}$ and $\boldsymbol{v}_{t}^{j}$ are the projections of the velocities of the interacting particles onto the plane whose normal is $\boldsymbol{d}$ (see Fig. 3). Note that this definition of the tangential direction implies that all the contacts $c$ composing an interaction share the same vector $\boldsymbol{t}$. The tangential overlap $\delta_{t}$ is the relative displacement of the interacting particles along the tangential direction. Then the force $\boldsymbol{F}$ of the interaction $\xi$ between two particles is calculated by adding the forces $f$ exerted at each contact $c$ :

$$
\boldsymbol{F}=\sum_{c \in \xi} \boldsymbol{f}^{c}
$$

The particle translations are integrated by means of a Leap-Frog algorithm. The rotations are integrated using the algorithm presented in Appendix A.

\section{Sample construction and shear test}

Ten monodisperse samples made up of 8000 spheroplates are built. The difference between these samples is their platyness, which varies from $\eta=0$ to 0.8 . The spheroradius $r=30 \mathrm{~nm}$ is the same for all samples, and the density $\rho$ is set to $2700 \mathrm{~kg} / \mathrm{m}^{3}$. The normal and tangential stiffnesses, $k_{n}$ and $k_{t}$ respectively, are set to $1.5 \times 10^{-3} \mathrm{~N} / \mu \mathrm{m}$. Note that the particle size, density, and stiffness are typical of fine grained soils [2-6]. As recommended in Ref. [17], the damping coefficients are calibrated in order for the restitution coefficient
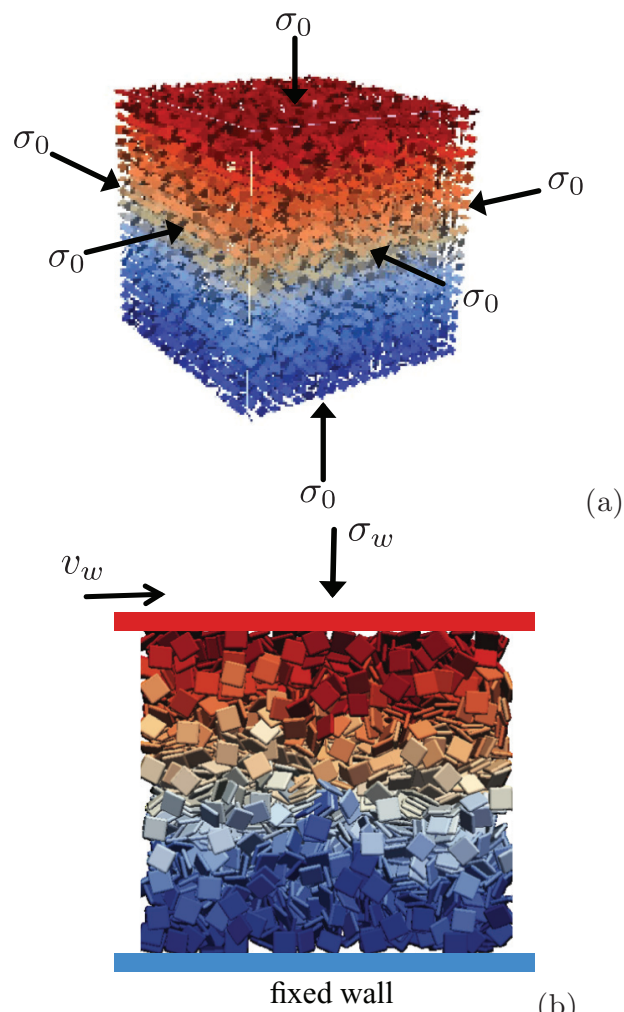

(b)

FIG. 4. (Color online) (a) Sample construction and isotropic compression. (b) Shear test.

in binary collisions between spheroplates to be around 0.6. However, it has been shown that this parameter has practically no effect in the rheology if the system is sheared quasistatically and if the particles are frictional; see Refs. [15,16]. The friction coefficient is set to $\mu=0.58 \simeq \tan \left(30^{\circ}\right)$, which is close to that of kaolinite clay [2-6].

Initially, the particles are placed at the nodes of a cubic grid of side $2 \sqrt{2} R$ and each of them is randomly oriented. These samples are isotropically compressed under a stress $\sigma_{0}$; see Fig. 4(a). Once the system attains static equilibrium, the lateral walls are removed and replaced by periodic boundaries. The samples are then sheared by imposing a constant velocity $v_{w}$ and a constant confining stress $\sigma_{w}$ to the upper wall [see Fig. 4(b)], allowing for the volume of the sample to vary during the test. The particles in contact with the walls are "glued" to them in order to avoid strain localization at the boundaries. In all simulations presented in this paper the gravity is set to zero in order to get homogeneous stress fields inside the packings.

As mentioned in Introduction, the focus of this work is on the quasistatic mechanical behavior in the steady state. Therefore, the samples are sheared up to a large cumulative shear strain $\gamma=x_{w} / y_{w} \simeq 2.5$, where $x_{w}$ is the horizontal displacement of the upper wall and $y_{w}$ is its vertical position. Figure 5 shows the shear stress ratio $\tau_{w} / \sigma_{w}$, where $\tau_{w}$ and $\sigma_{w}$ are, respectively, the shear and normal stresses at the moving wall, as a function of $\gamma$, for all values of the platyness $\eta$. We see that all packings reach the steady state, since, at the end of the shear test, $\tau_{w} / \sigma_{w}$ fluctuates around a mean value.

Figure 6 shows the profiles of the time-averaged horizontal velocity $\left\langle v_{x}\right\rangle$ of the particles as a function of their normalized 


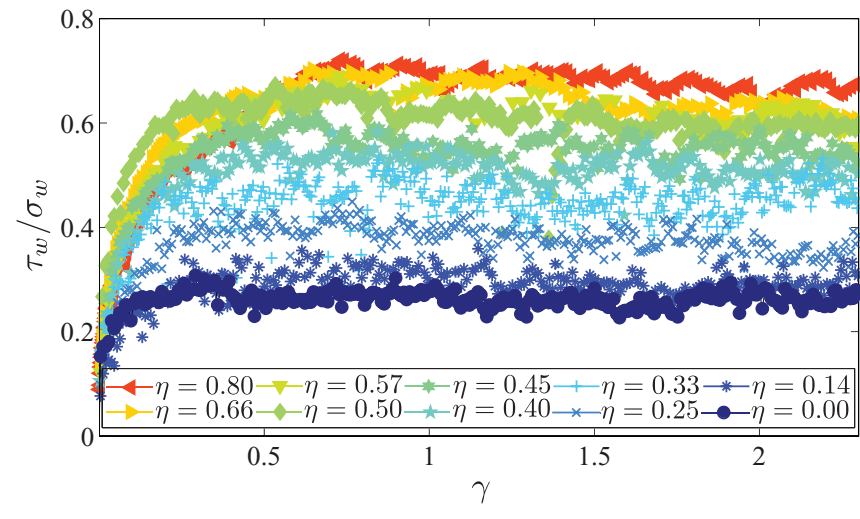

FIG. 5. (Color online) Shear stress ratio $\tau_{w} / \sigma_{w}$ as a function of the shear strain $\gamma$ for all values of $\eta$.

vertical position $y / y_{w}$, for all values of the platyness $\eta$. We see that the packing is sheared in the whole bulk with no sign of strain localization in the steady state.

The quasistatic feature of the simple shear tests and the stiffness of the particles can be evaluated, respectively, with two dimensionless parameters $[15,16]$ : the inertia parameter $I$ and the dimensionless stiffness $\kappa$ defined as

$$
I=\dot{\gamma} r \sqrt{\frac{\rho}{\sigma_{w}}} \quad \text { and } \quad \kappa=\frac{k_{n}}{\sigma_{w} r}
$$

where $\dot{\gamma}$ is the shear rate. A sheared system is in a quasistatic regime if $I \ll 1$ and the contact interactions can be considered to be in the rigid limit if $\kappa$ is large. In all our tests, we have $I \approx 10^{-3}$ and $\kappa \geqslant 10^{3}$. Video samples of some of the simulations analyzed in this paper can be found at www.cgpgateway.org/ref021.

\section{MECHANICAL BEHAVIOR AT THE MACROSCALE}

In this section we analyze both stresses and strains in the steady state, in terms of shear strength and packing fraction. All presented quantities correspond to the average over the last $40 \%$ of cumulative shear strain (i.e., from $\gamma=1.5$ to 2.5 ).

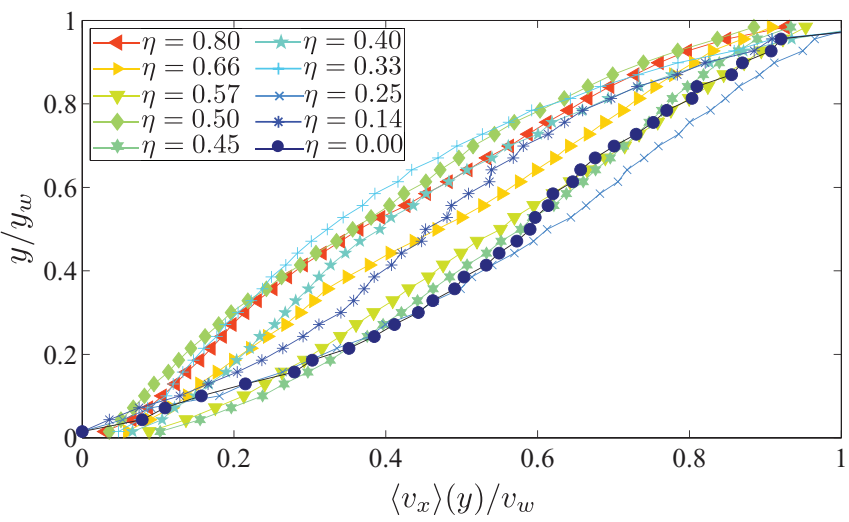

FIG. 6. (Color online) Profiles of the time averaged horizontal velocity $\left\langle v_{x}\right\rangle$ of the particles as a function of their normalized vertical position $y / y_{w}$ for all values of the platyness $\eta$.

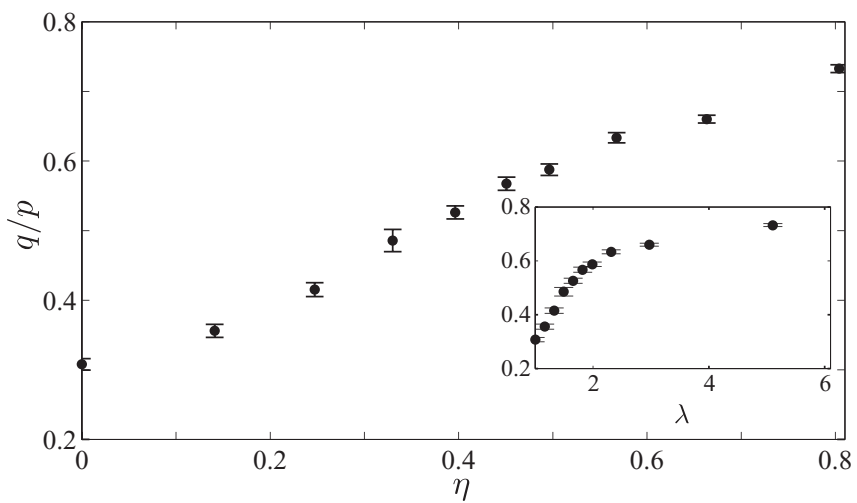

FIG. 7. Shear strength $q / p$ as a function of the platyness $\eta$. The inset shows $q / p$ as a function of the aspect ratio $\lambda$. Error bars indicate the standard deviation.

\section{A. Shear strength}

The shear strength was calculated from the stress tensor $\sigma$, defined by

$$
\boldsymbol{\sigma}=\frac{1}{V} \sum_{\xi \in V} F_{\alpha}^{\xi} \ell_{\beta}^{\xi}
$$

where $V$ is the volume containing the interactions $\xi, \boldsymbol{F}$ is the interaction force, $\ell$ is the branch vector, i.e., the vector joining the centroids of the interacting particles, and $\alpha$ and $\beta$ denote the components in the reference frame. The partial mean stress $p=\left(\sigma_{1}+\sigma_{3}\right) / 2$ and partial deviatoric stress $q=\left(\sigma_{1}-\sigma_{3}\right) / 2$ are then calculated from the principal stresses $\sigma_{1}$ and $\sigma_{3}$. We exclude the intermediate principal stress $\sigma_{2}$, since the simple shear test here is under plane strain conditions, the strain being zero in the intermediate direction.

The shear strength of the material can be expressed by the stress ratio $q / p$. Figure 7 shows $q / p$ as a function of the platyness $\eta$; the inset shows $q / p$ as a function of the aspect ratio $\lambda$. We can see that $q / p$ increases with $\eta$, approximately from 0.3 to 0.7 . This means that, as the particle platyness increases, the material's shear strength also increases.

\section{B. Packing fraction}

The packing fraction is defined as $v=V_{p} / V$, where $V_{p}$ is the volume occupied by the particles and $V$ is the total volume. Figure 8 shows $v$ as a function of platyness $\eta$, the inset shows $v$ as a function of the aspect ratio $\lambda$. We can see that $v$ first increases with $\eta$, reaches a maximum at $\eta \simeq 0.3$, and then declines as $\eta$ further increases. As later discussed in Sec. V, this is commonly obtained when varying particle shape.

\section{MICROSTRUCTURE}

In this section we analyze the microstructure of the samples, in terms of particle orientations, connectivity, fabric, and force networks. Again, all presented quantities correspond to the average over the last $40 \%$ of cumulative shear strain (i.e., from $\gamma=1.5$ to 2.5 ). 


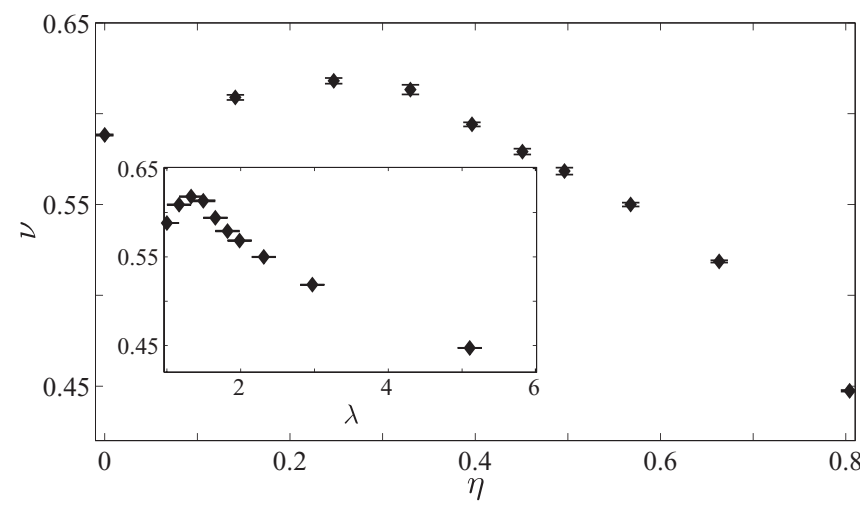

FIG. 8. Packing fraction $v$ as a function of the platyness $\eta$. The inset shows $v$ as a function of the aspect ratio $\lambda$. Error bars indicate the standard deviation.

\section{A. Particle orientations}

The particle orientation is defined as the orientation of the vector $\boldsymbol{m}$, normal to the particle face; see Fig. 9(a). The distribution of particle orientations can be represented by the probability density function $P_{m}(\Omega)$ of particles whose vector $\boldsymbol{m}$ is along the solid angle $\Omega=(\theta, \phi)$; see Fig. 9(b). Figure 10 shows the distributions $P_{m}(\Omega)$ for three samples composed of particles with platyness $\eta=0.33,0.5$, and 0.8 , at $\gamma=2$. Because of the planar symmetry of our simple shear tests, we consider the restriction of $P_{m}(\Omega)$ to the $x y$ plane, i.e., the function $P_{m}(\theta)$, which can be approximated by its lowest order Fourier expansion:

$$
P_{m}(\theta) \simeq 1 / \pi\left[1+a_{m} \cos 2\left(\theta-\theta_{m}\right)\right],
$$

where $a_{m}$ is the anisotropy of particle orientations and $\theta_{m}$ is the principal direction of $P_{m}(\theta)$. Even though the parameters $a_{m}$ and $\theta_{m}$ can be calculated by fitting the measured values of $P_{m}(\theta)$ to the approximation presented in Eq. (8), in practice it is more convenient to calculate these parameters using the nematic tensor defined by

$$
\mathbf{M}=\frac{1}{N_{p}} \sum_{p \in V} m_{\alpha}^{p} m_{\beta}^{p},
$$

where $N_{p}$ is the number of particles $p$ in the volume $V, \boldsymbol{m}$ is the particle orientation, and $\alpha$ and $\beta$ denote the components in the reference frame. The anisotropy of particle orientations is $a_{m}=2\left(\mathrm{M}_{1}-\mathrm{M}_{3}\right)$, and the principal direction $\theta_{m}$ is that of the first eigenvalue of $\mathbf{M}$.
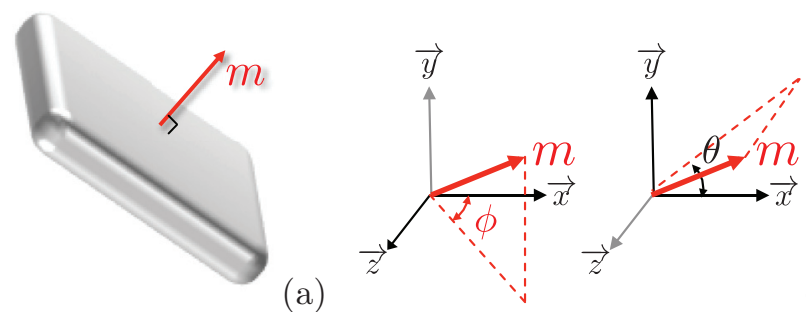

(b)

FIG. 9. (Color online) (a) Definition of the particle orientation vector $\boldsymbol{m}$ (normal to the particles face). (b) Orientation angles $\theta$ and $\phi$ of the vector $\boldsymbol{m}$ at the reference system.
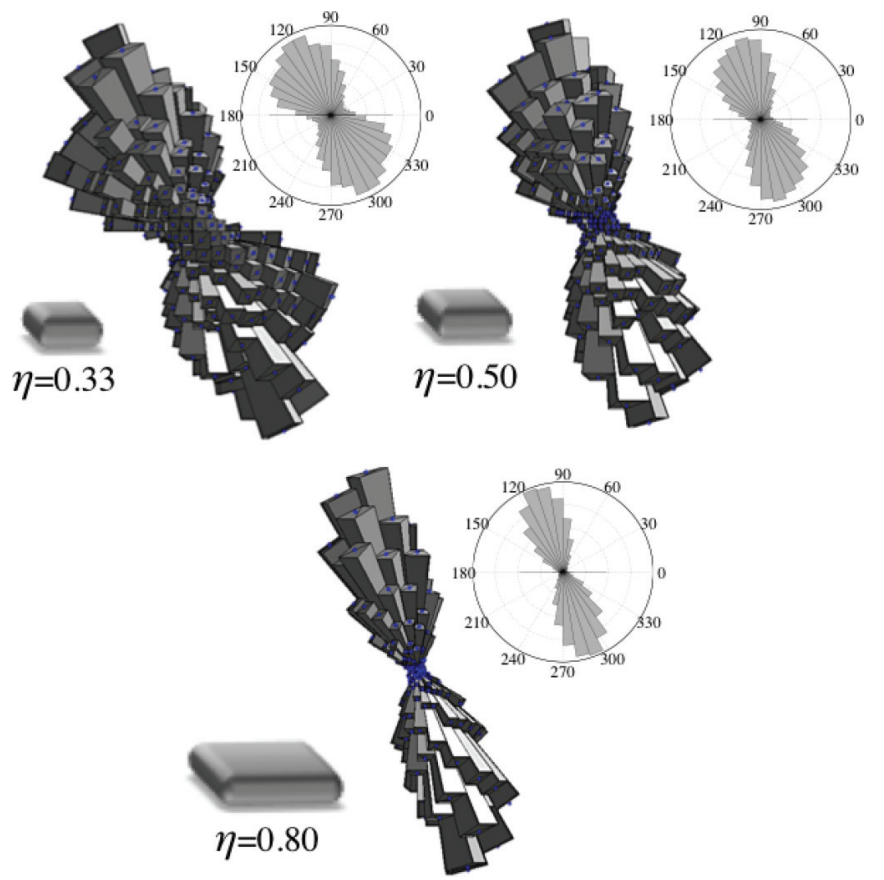

FIG. 10. (Color online) Probability density functions $P_{m}(\Omega)$ of particle orientations and their restrictions $P_{m}(\theta)$ to the $x y$ plane for three samples composed of particles of platyness $\eta=0.33,0.5$, and 0.8 , at $\gamma=2$.

Figure 11 shows the anisotropy of particle orientations $a_{m}$ and the principal direction $\theta_{m}$ as functions of platyness $\eta$. We can see that $a_{m}$ increases from 0 to approximately 0.6 , showing that, as platyness increases, the number of particles aligning their faces along a particular direction increases. Following an opposite trend, $\theta_{m}$ decreases with $\eta$, approximately from $120^{\circ}$ to $105^{\circ}$, showing that, as platyness increases, the direction along which these particles align gradually approaches the vertical direction. Note that this type of ordering appears even in the samples composed of particles with very low values of $\eta$, e.g., for $\eta=0.14$, whose shape deviates only slightly from that of a sphere. This ordering phenomenon has a strong influence on the response of the material, as it will be shown in the following sections and as explained in detail in Sec. V.

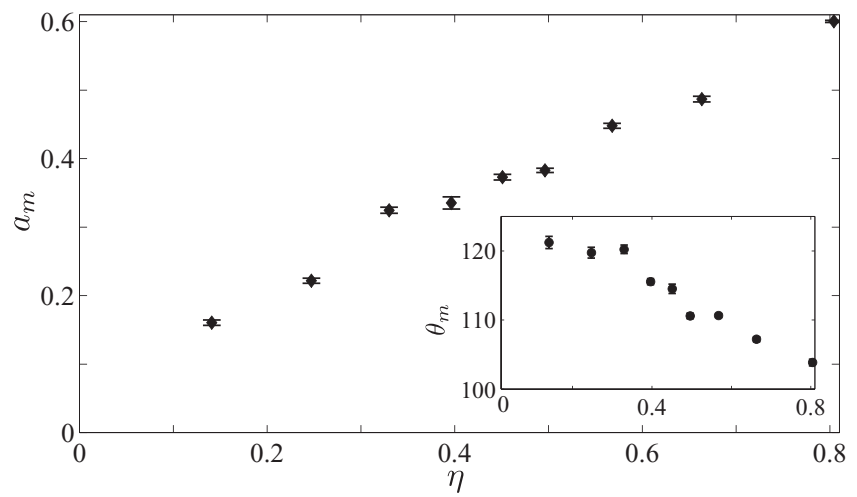

FIG. 11. Anisotropy of particle orientations $a_{m}$ as a function of the platyness $\eta$. The inset shows the principal direction $\theta_{m}$ as a function of $\eta$. Error bars indicate the standard deviation. 

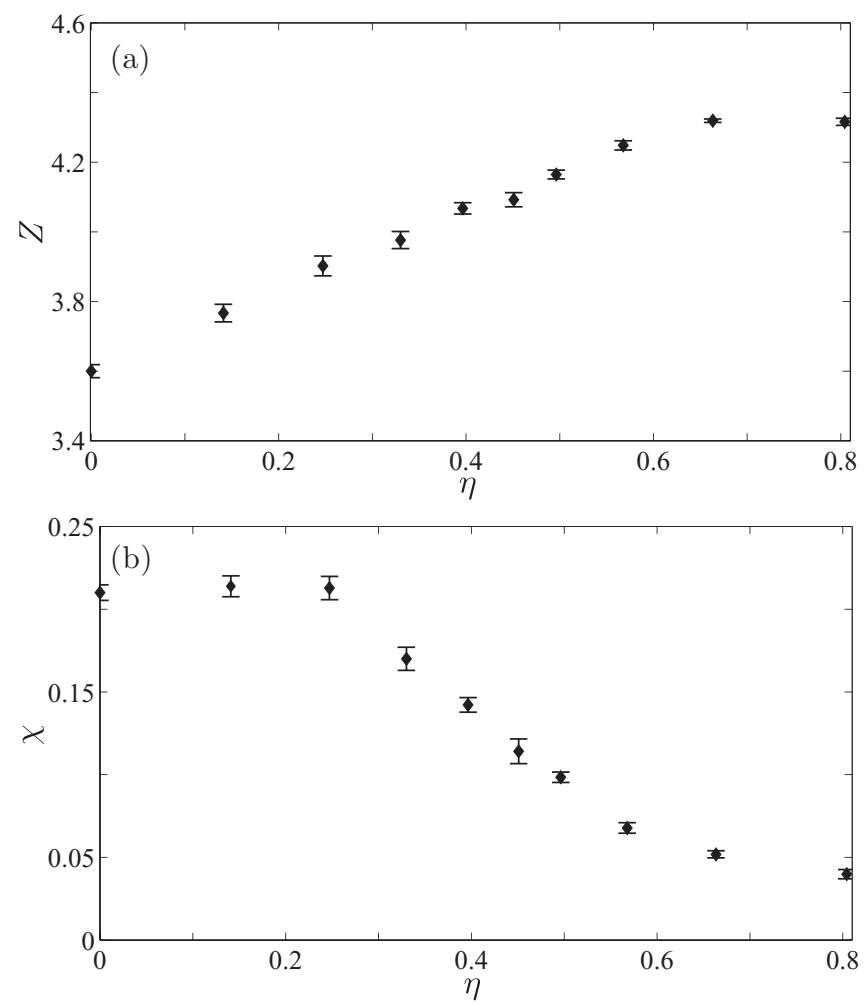

FIG. 12. (a) Coordination number $Z$ as a function of the platyness $\eta$. (b) Proportion $\chi$ of floating particles as a function of $\eta$. Error bars indicate the standard deviation.

\section{B. Connectivity and fabric of the interactions network}

Figure 12(a) shows the coordination number $Z$ as a function of the platyness $\eta$. Note that $Z$ is the mean number of interactions per particle, where an interaction can comprise several contacts; see Sec. II A. We can see that $Z$ increases with $\eta$, approximately from 3.6 to 4.3 , and stabilizes for the larger values of $\eta$. This shows that, as platyness increases, the particle connectivity also increases. This is a counterintuitive result, since, as was shown in Sec. III B, these very connected samples are also the loosest ones.

Figure 12(b) shows the proportion $\chi$ of floating particles as a function of platyness $\eta$. These are particles that have zero or one interaction and, thus, do not take part in the force carrying structure. We can see that $\chi$ decreases with $\eta$, approximately from 0.20 to 0.05 . This shows that, as platyness increases, the number of particles participating in the force-carrying network also increases. This is consistent with the increase in coordination as a function of platyness.

Let us now define an interaction frame, associated to each interaction between two interacting particles, as $(\boldsymbol{n}, \boldsymbol{t})$; see Sec. II B for a definition of $\boldsymbol{n}$ and $\boldsymbol{t}$. The interaction frame is such that, in an interaction comprising several contacts, all of these contacts share the same normal and tangental directions. The fabric of the interaction network can be represented by the probability density function $P_{n}(\Omega)$ of interactions along $\Omega$. Figure 13 shows $P_{n}(\Omega)$ for four samples composed of particles with platyness $\eta=0,0.33,0.5$, and 0.8 , at $\gamma=2$. Again, because of the planar symmetry, we are interested in the restriction $P_{n}(\theta)$ in the $x y$ plane. It can be approximated
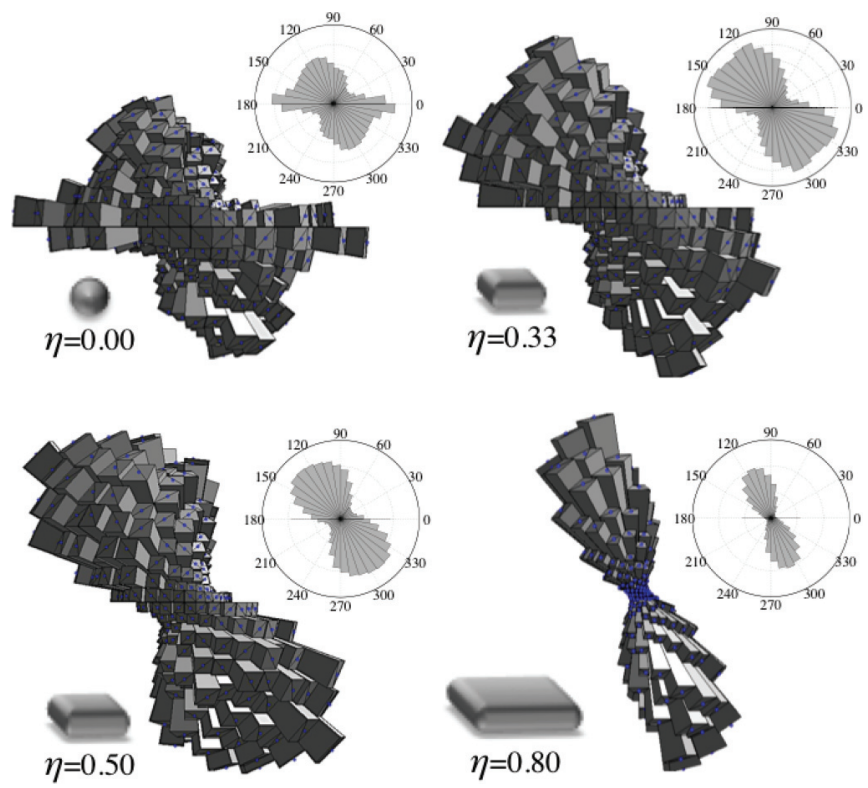

FIG. 13. (Color online) Probability density functions $P_{n}(\Omega)$ of interaction orientations and their restrictions $P_{n}(\theta)$ to the shear $x y$ plane for four samples composed of particles with platyness $\eta=0$, $0.33,0.5$, and 0.8 , at shear strain $\gamma=2$.

by its lowest-order Fourier expansion:

$$
P_{n}(\theta) \simeq 1 / \pi\left[1+a_{n} \cos 2\left(\theta-\theta_{n}\right)\right],
$$

where $a_{n}$ represents the leading anisotropy of interaction orientations and $\theta_{n}$ is the priviledged direction of $\theta$; see Fig. 13. Again, the parameters $a_{n}$ and $\theta_{n}$ can be calculated by fitting the measured values of $P_{n}(\theta)$ to the approximation presented in Eq. (10), but in practice it is more convenient to calculate these parameters using the fabric tensor, defined as

$$
\mathbf{F}_{\alpha \beta}=\frac{1}{N_{\xi}} \sum_{\xi \in V} n_{\alpha}^{\xi} n_{\beta}^{\xi},
$$

where $N_{\xi}$ is the number of interactions $\xi$ in the volume $V, \boldsymbol{n}$ is the normal vector, and $\alpha$ and $\beta$ denote the components in the reference frame. The anisotropy of interaction orientations is $a_{n}=2\left(\mathrm{~F}_{1}-\mathrm{F}_{3}\right)$, and the principal direction $\theta_{n}$ is the direction of the first eigenvalue of $\mathbf{F}$.

Figure 14 shows the anisotropy of interaction orientations $a_{n}$ and the principal direction $\theta_{n}$ as functions of the platyness $\eta$. We see that $a_{n}$ increases with $\eta$, approximately from 0.3 to 1.2 , showing that, as platyness increases, the number of interactions aligning along a particular direction also increases. Then, as observed for $\theta_{m}, \theta_{n}$ decreases with $\eta$, approximately from $135^{\circ}$ to $110^{\circ}$. In other words, as platyness increases, the direction along which these interactions align gradually approaches the vertical direction. The similarities between the parameters describing the distributions $P_{m}(\theta)$ and $P_{n}(\theta)$ are to be expected, since, by construction, the vectors $\boldsymbol{m}$ and $\boldsymbol{n}$ are correlated in interactions involving the particle faces, and, as the particle platyness increases, the proportion of this type of interactions is expected to increase.

Other descriptors of the fabric can be calculated using the branch vector $\ell$, which can be decomposed in the normal and 


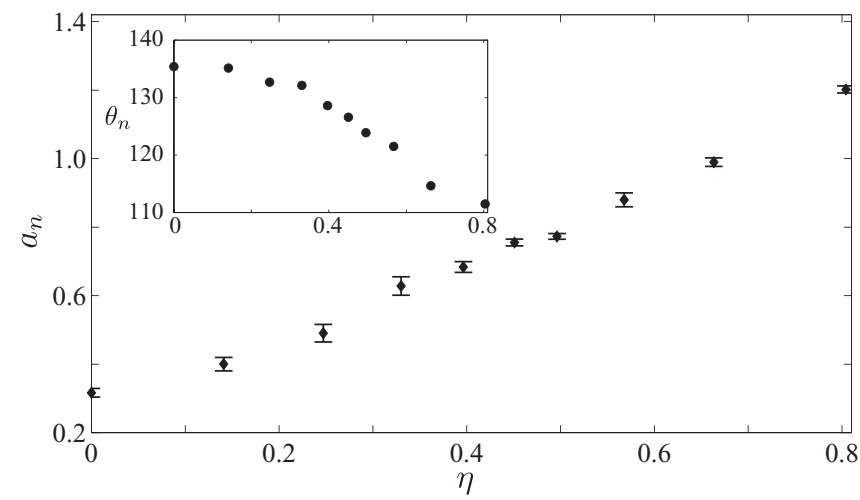

FIG. 14. Anisotropy of interaction orientations $a_{n}$ as a function of the platyness $\eta$. The inset shows the principal direction $\theta_{n}$ as a function of $\eta$. Error bars represent the standard deviation.

tangential directions:

$$
\ell=\ell_{n} n+\ell_{t} \boldsymbol{t},
$$

where $\ell_{n}$ and $\ell_{t}$ are, respectively, the normal and tangential branch lengths, as shown in Fig. 15.

The angular distributions of branch lengths can be represented by $\left\langle\ell_{n}\right\rangle(\Omega)$ and $\left\langle\ell_{t}\right\rangle(\Omega)$, which are, respectively, the average branch lengths in the normal and tangential directions. By construction, $\ell_{n}$ is always positive whereas $\ell_{t}$ can take positive or negative values. Figure 16 shows $\left\langle\ell_{n}\right\rangle(\Omega)$ and $\left\langle\ell_{t}\right\rangle(\Omega)$ for four samples composed of particles with platyness $\eta=0,0.33,0.5$, and 0.8 , at shear strain $\gamma=2$. Again, because of the symmetry of our simple shear tests, we are interested in the restrictions $\left\langle\ell_{n}\right\rangle(\theta)$ and $\left\langle\ell_{t}\right\rangle(\theta)$, which can be approximated by their lowest order Fourier expansions:

$$
\begin{gathered}
\left\langle\ell_{n}\right\rangle(\theta)=\left\langle\ell_{n}\right\rangle\left[1+a_{\ell n} \cos 2\left(\theta-\theta_{\ell n}\right)\right], \\
\left\langle\ell_{t}\right\rangle(\theta)=\left\langle\ell_{n}\right\rangle a_{\ell t} \sin 2\left(\theta-\theta_{\ell t}\right),
\end{gathered}
$$

where $a_{l n}$ and $a_{l t}$ are, respectively, the anisotropies of normal and tangential branch lengths, and $\theta_{l n} \simeq \theta_{l t}$ are, respectively, the priviledged directions of $\left\langle\ell_{n}\right\rangle(\theta)$ and $\left\langle\ell_{t}\right\rangle(\theta)$. Again, the parameters $a_{l n}, a_{l t}$, and $\theta_{l n}$ can be calculated by fitting the measured values of $\left\langle\ell_{n}\right\rangle(\theta)$ and $\left\langle\ell_{t}\right\rangle(\theta)$ to the approximations presented in Eqs. (13) and (14), but in practice it is more convenient to calculate these parameters from the following

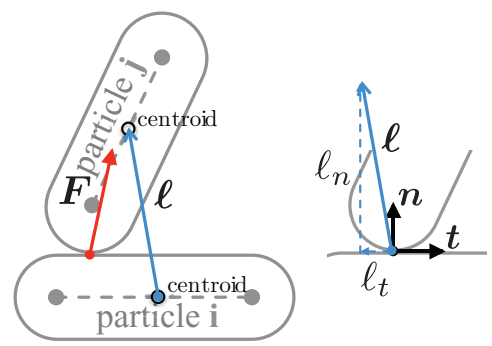

FIG. 15. (Color online) Definition of the vectors $\ell_{n}$ and $\ell_{t}$ using the normal $\boldsymbol{n}$ and tangential $\boldsymbol{t}$ directions of a interaction between two particles.

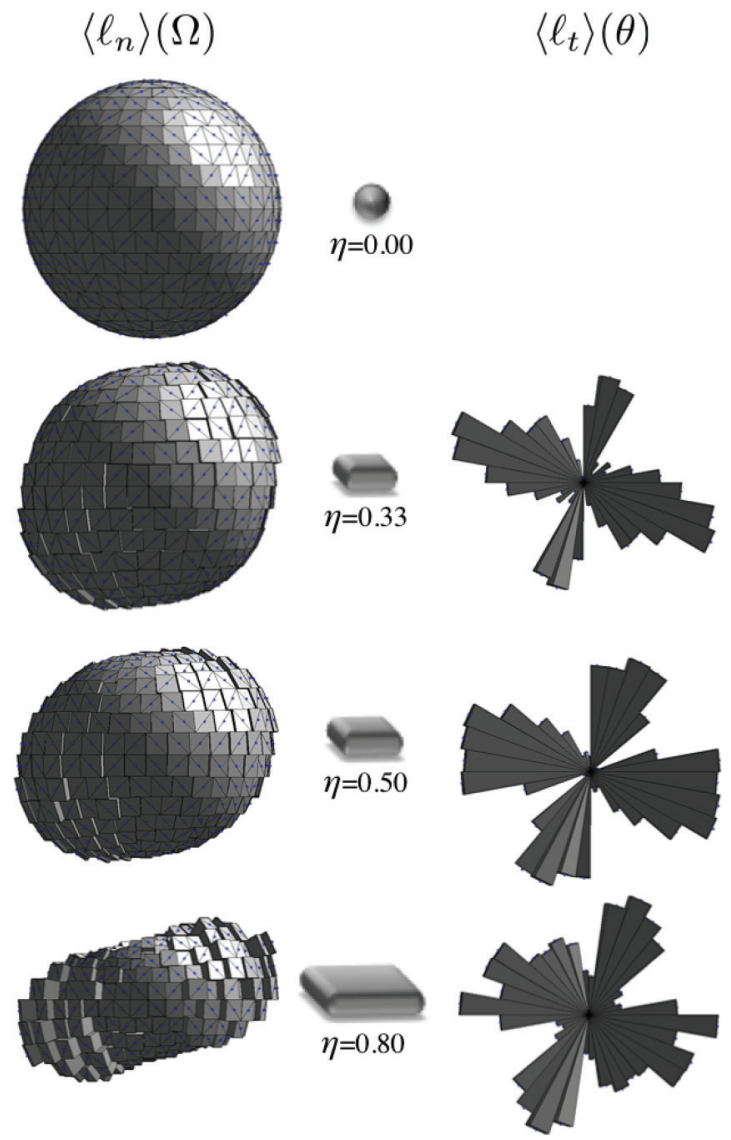

FIG. 16. (Color online) Distributions $\left\langle\ell_{n}\right\rangle(\Omega)$ and $\left\langle\ell_{t}\right\rangle(\theta)$ of average branch lengths in the normal and tangential directions, respectively, for four samples composed of particles with platyness $\eta=0,0.33,0.5$, and 0.8 , at a shear strain $\gamma=2$.

tensors:

$$
\begin{gathered}
\chi_{\alpha \beta}^{l n}=\frac{1}{N_{\xi}} \sum_{\xi \in V} \ell_{n}^{\xi} n_{\alpha}^{\xi} n_{\beta}^{\xi}, \\
\chi_{\alpha \beta}^{l t}=\frac{1}{N_{\xi}} \sum_{\xi \in V} \ell_{t}^{\xi} n_{\alpha}^{\xi} t_{\beta}^{\xi}, \\
\chi^{l}=\chi^{l n}+\chi^{l t},
\end{gathered}
$$

where $N_{\xi}$ is the number of interactions $\xi$ in a volume $V, \ell$ is the branch vector, $\boldsymbol{n}$ is the normal vector, $\boldsymbol{t}$ is the tangential vector, and $\alpha$ and $\beta$ denote de components in the reference frame. Note that, by construction, we have $\chi^{l t}=0$. From Eq. (17), assuming that $\theta_{l n} \simeq \theta_{l t} \simeq \theta_{n}$, the following relations are easily obtained:

$$
\begin{aligned}
& a_{l n}=2\left(\chi_{1}^{l n}-\chi_{3}^{l n}\right) /\left(\chi_{1}^{l n}+\chi_{3}^{l n}\right)-a_{c}, \\
& a_{l t}=2\left(\chi_{1}^{l}-\chi_{3}^{l}\right) /\left(\chi_{1}^{l}+\chi_{3}^{l}\right)-a_{c}-a_{l n} .
\end{aligned}
$$

Figure 17 shows the anisotropies of normal and tangential branch lengths, $a_{l n}$ and $a_{l t}$ respectively, as well as the principal direction $\theta_{l n}$, as functions of the platyness $\eta$. First, we can see that $a_{l n}$ decreases with $\eta$, from 0 to approximately -0.2 . This happens because, as platyness increases, the interactions oriented along the principal orientation defined in the previous section tend to be predominantly face-face interactions, 


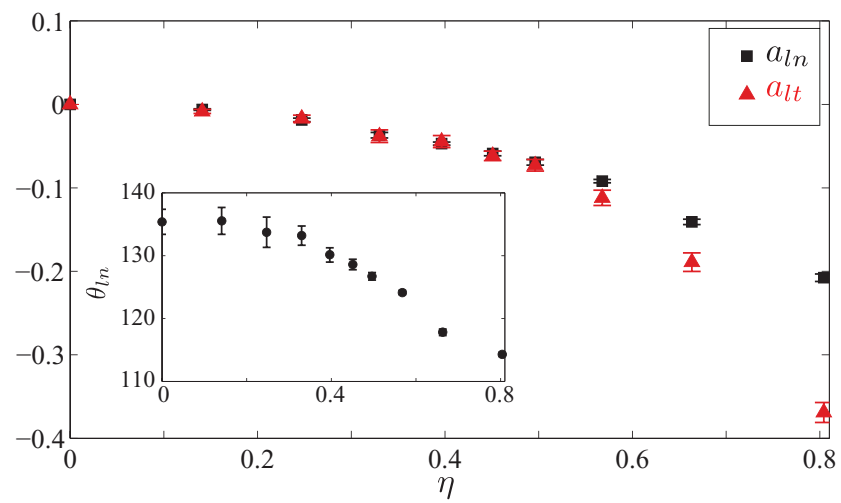

FIG. 17. (Color online) Anisotropies of normal (black) and tangential (red) branch lengths, $a_{l n}$ and $a_{l t}$, respectively, as functions of the platyness $\eta$. The inset shows the principal direction $\theta_{l n}$ as a function of $\eta$. Error bars represent the standard deviation.

characterized by short branch lengths, while the interactions oriented orthogonally to these principal orientation tend to be predominantly edge-edge interactions characterized by long branch lengths. Second, we see that $a_{l t}$ decreases with $\eta$, from 0 to approximately -0.4 . This shows that, as platyness increases, the possibility of having tangential branch lengths also increases, as the vectors $\boldsymbol{n}$ and $\boldsymbol{\ell}$ can have different orientations. Third, as previously observed for $\theta_{m}$ and $\theta_{n}$, $\theta_{l n}$ decreases with $\eta$, approximately from $135^{\circ}$ to $110^{\circ}$. This means that, as platyness increases, the direction along which the face-face interactions are predominantly forming gradually approaches the vertical direction.

\section{Interaction forces}

As mentioned in Sec. II B, the interaction force $\boldsymbol{F}$ between two particles is calculated by adding the forces $f$ exerted at each contact point. As $\boldsymbol{f}, \boldsymbol{F}$ can be decomposed in the normal and tangential directions:

$$
\boldsymbol{F}=F_{n} \boldsymbol{n}+F_{t} \boldsymbol{t} .
$$

The angular distributions of interaction forces can be represented by $\left\langle F_{n}\right\rangle(\Omega)$ and $\left\langle F_{t}\right\rangle(\Omega)$, which are, respectively, the average forces in the normal and tangential directions, as shown in Fig. 18.

By construction, $F_{n}$ is always positive whereas $F_{t}$ can take positive or negative values. Figure 19 shows $\left\langle F_{n}\right\rangle(\Omega)$ and $\left\langle F_{t}\right\rangle(\Omega)$ for four samples composed of particles with platyness $\eta=0,0.33,0.5$, and 0.8 , at shear strain $\gamma=2$. Here

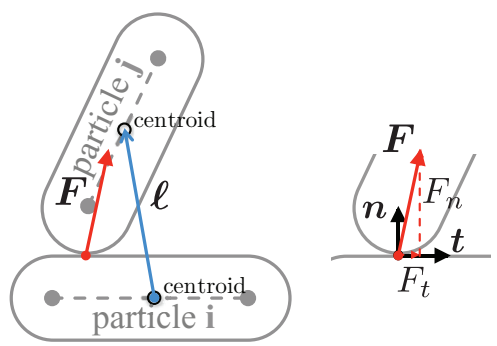

FIG. 18. (Color online) Definition of the vectors $F_{n}$ and $F_{t}$ using the normal $\boldsymbol{n}$ and tangential $\boldsymbol{t}$ directions of a interaction between two particles.

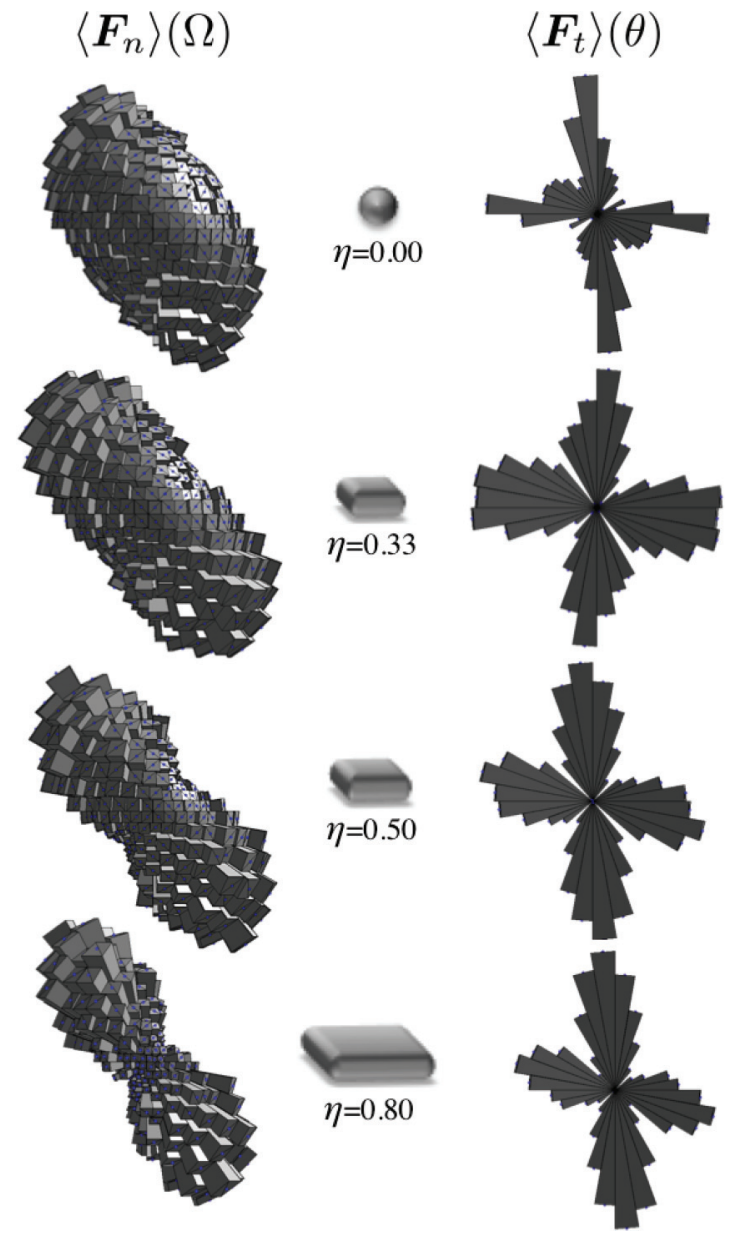

FIG. 19. (Color online) Distributions $\left\langle\boldsymbol{F}_{n}\right\rangle(\Omega)$ and $\left\langle\boldsymbol{F}_{t}\right\rangle(\theta)$ of average forces in the normal and tangential directions, respectively, for four samples composed of particles with platyness $\eta=0,0.33$, 0.5 , and 0.8 , at shear strain $\gamma=2$.

again, because of planar symmetry, we consider the restrictions $\left\langle F_{n}\right\rangle(\theta)$ and $\left\langle F_{t}\right\rangle(\theta)$ in the shear plane. These functions can be approximated by their lowest order Fourier expansions:

$$
\begin{gathered}
\left\langle F_{n}\right\rangle(\theta)=\left\langle F_{n}\right\rangle\left[1+a_{f n} \cos 2\left(\theta-\theta_{f n}\right)\right], \\
\left\langle F_{t}\right\rangle(\theta)=\left\langle F_{n}\right\rangle a_{f t} \sin 2\left(\theta-\theta_{f t}\right),
\end{gathered}
$$

where $a_{f n}$ and $a_{f t}$ are, respectively, the anisotropies of normal and tangential forces, and $\theta_{f n} \simeq \theta_{f t}$ are, respectively, the principal directions of $\left\langle F_{n}\right\rangle(\theta)$ and $\left\langle F_{t}\right\rangle(\theta)$. The parameters $a_{f n}, a_{f t}$, and $\theta_{f n}$ can be calculated by fitting the measured values of $\left\langle F_{n}\right\rangle(\theta)$ and $\left\langle F_{t}\right\rangle(\theta)$ to the approximations presented in Eqs. (21) and (22), but it is more convenient to calculate these parameters using the following tensors:

$$
\begin{aligned}
\chi_{\alpha \beta}^{f n} & =\frac{1}{N_{\xi}} \sum_{\xi \in V} F_{n}^{\xi} n_{\alpha}^{\xi} n_{\beta}^{\xi}, \\
\chi_{\alpha \beta}^{f t} & =\frac{1}{N_{\xi}} \sum_{\xi \in V} F_{t}^{\xi} n_{\alpha}^{\xi} t_{\beta}^{\xi}, \\
\chi^{f} & =\chi^{f n}+\chi^{f t},
\end{aligned}
$$




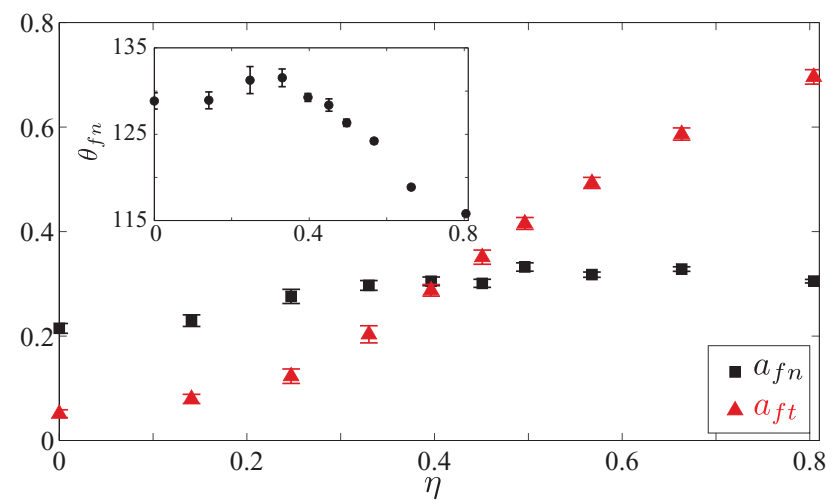

FIG. 20. (Color online) Anisotropies of normal (black) and tangential (red) interaction forces, $a_{f n}$ and $a_{f t}$ respectively, as functions of the platyness $\eta$. The inset shows the principal direction $\theta_{f n}$ as a function of $\eta$. Error bars represent the standard deviation.

where $N_{\xi}$ is the number of interactions $\xi$ in a volume $V, \boldsymbol{F}$ is the interaction force, $\boldsymbol{n}$ is the normal vector, and $\alpha$ and $\beta$ denote de components in the reference frame. Note that, by construction, we have $\chi^{f t}=0$. From Eq. (25), assuming that $\theta_{l n} \simeq \theta_{l t} \simeq \theta_{n}$, the following relations are easily obtained:

$$
\begin{gathered}
a_{f n}=2\left(\chi_{1}^{f n}-\chi_{3}^{f n}\right) /\left(\chi_{1}^{f n}+\chi_{3}^{f n}\right)-a_{c}, \\
a_{f t}=2\left(\chi_{1}^{f}-\chi_{3}^{f}\right) /\left(\chi_{1}^{f}+\chi_{3}^{f}\right)-a_{c}-a_{f n} .
\end{gathered}
$$

Figure 20 shows the anisotropies of normal and tangential interaction forces, $a_{f n}$ and $a_{f t}$ respectively, as well as their principal direction $\theta_{f n}$, as functions of the platyness $\eta$. First, we see that $a_{f n}$ increases slowly with $\eta$, approximately from 0.2 to 0.3 . This shows that the particle platyness has little effect on the magnitude of the normal forces transmitted between particles. Second, $a_{f t}$ increases with $\eta$, approximately from 0 to 0.7 . This means that, as platyness increases, the magnitude of the tangential forces transmitted between particles also increases. Third, as observed for $\theta_{m}, \theta_{n}$ and $\theta_{l n}, \theta_{f n}$ decreases with $\eta$, approximately from $130^{\circ}$ to $115^{\circ}$, showing that, as platyness increases, the direction along which the largest normal forces are transmitted, gradually approaches the vertical direction; the direction along which the largest tangential forces are transmitted is shifted by approximately $45^{\circ}$.

The slow increase of the anisotropy of normal interaction forces $a_{f n}$ and the fast increase of the anisotropy of tangential interaction forces $a_{f t}$ with platyness $\eta$ suggest that, as platyness increases, the stability of the interactions depends more and more strongly on friction forces. At the lowest order, this can be quantified by considering the proportion $K_{s}$ of "sliding" interactions (i.e., those interactions in which $F_{t} / F_{n} \simeq \mu$ ). Figure 21 shows $K_{s}$ as a function of $\eta$. It can be seen that $K_{s}$ increases with $\eta$, approximately from 0.1 to 0.4 . Hence, as platyness increases, the level of friction mobilization, which reflects de dependence of the mechanical stability of the material on friction forces, also increases. The relationship between particle shape and friction mobilization seems to be a robust feature of granular materials composed of complex-shaped particles, as shown in Sec. V.

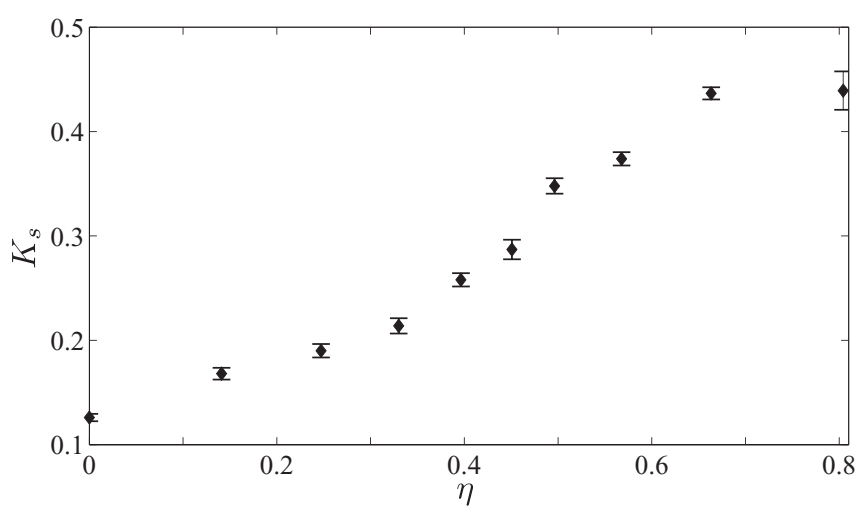

FIG. 21. Proportion of sliding interactions $K_{s}$ as a function of the platyness $\eta$. Error bars indicate the standard deviation.

\section{Micromechanical origins of the shear strength}

Throughout this section, we introduced a series of anisotropy parameters (anisotropies of particle orientations $a_{m}$, interaction orientations $a_{n}$, normal branch lengths $a_{l n}$, tangential branch lengths $a_{l t}$, normal interaction forces $a_{f n}$, and tangential interaction forces $a_{f t}$ ). Each of these anisotropies encodes part of the anisotropic structure of the packings. In fact, as shown in Ref. [18], these anisotropies sum up to produce the shear strength of the material, as expressed by the following relation based on the assumption that the cross-products of the anisotropies can be neglected:

$$
\begin{aligned}
q / p \simeq & \frac{1}{2}\left[a_{c} \cos 2\left(\theta_{n}-\theta_{\sigma}\right)+a_{l n} \cos 2\left(\theta_{l n}-\theta_{\sigma}\right)\right. \\
& +a_{l t} \cos 2\left(\theta_{l t}-\theta_{\sigma}\right)+a_{f n} \cos 2\left(\theta_{f n}-\theta_{\sigma}\right) \\
& \left.+a_{f t} \cos 2\left(\theta_{f t}-\theta_{\sigma}\right)\right] .
\end{aligned}
$$

This is a useful decomposition, since it allows for exploring the different micromechanical origins of the shear strength $q / p$. Figure 22 shows the shear strength $q / p$, both measured in the shear tests and predicted from Eq. (28), as functions of the platyness $\eta$. We see that this expression approximates well the shear strength, especially at the low values of $\eta(\eta \lesssim 0.3)$. For the large values of $\eta$, this approximation overestimates the shear strength indicating that the assumptions involved in the development of Eq. (28) gradually lose their validity.

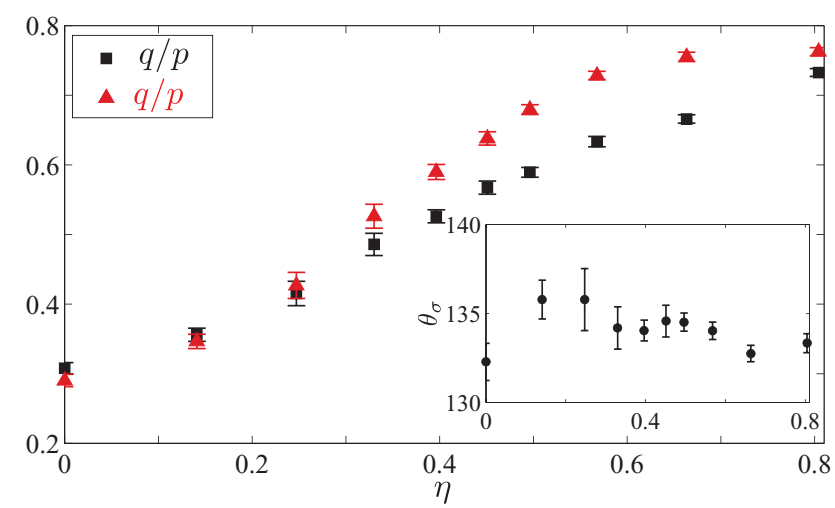

FIG. 22. (Color online) Shear strength from the data (squares) and its harmonic approximation (triangles) as a function of the platyness $\eta$. 
Finally, from Figs. 14 to 20, we can deduce that the increase of the shear strength with the platyness is mainly a consequence of the increase of the anisotropies of the interaction orientations and tangential interaction forces. This observation implies that the large shear strength of those materials composed of the most platy particles has its micromechanical origin in the orientation of the particles, which determines the direction of interaction normals, as well as in the mobilization of frictional forces, which can be approximated by the ratio $a_{f t} / a_{f n}$.

\section{SUMMARY AND DISCUSSION}

In summary, by means of discrete element simulations, we investigated the mechanical behavior and microstructure of granular materials made up of platy particles. The particles are three-dimensional square plates, approximated as spheropolyhedra. Several monodisperse samples of 8000 particles are built. The difference between these samples is the particle platyness, which is varied systematically from 0 , corresponding to spherical particles, to 0.8 , corresponding to particles which are 5 times larger than they are thick. These samples are sheared in the quasistatic limit up to a large shear deformation, in order to analyze their behavior in the steady state, also called "critical state" in soil mechanics. We analyzed the mechanical behavior of the material at the macroscale, in terms of shear strength and packing fraction, as well as its microstructure, in terms of particle orientations, connectivity, fabric, and forces networks.

We found that the mechanical behavior at the macroscale is strongly dependent on the particle platyness. First, the shear strength increases with platyness. This positive correlation between the particles shape and shear strength seems to be a robust feature in granular media, as it has been shown using experiments [19-21], simulations of two-dimensional angular particles [22-27], elongated particles [26-30], and nonconvex $[26,30,31]$ particles, as well as three-dimensional simulations $[8,32]$. Secondly, the relationship between the packing fraction and platyness is not monotonic. In fact, the packing fraction first increases with platyness up to a peak value, and then declines as platyness further increases. In other words, there is an optimum platyness for which the packing is found at a maximum packing fraction. This nonmonotonic behavior also seems to be a robust feature in granular media. Indeed, this relationship is reminiscent of that observed numerically for angular particles [26,30,33], elongated particles [26,27,29], and nonconvex particles [26,30,31] in two dimensions, as well as for ellipsoidal particles in three dimensions [34].

From the micromechanical point of view, we found that the principal phenomenon underlying the increase of shear strength is the alignment of particle faces along a particular direction. Curiously, this type of ordering emerges even in the samples composed of particles with very low platyness, whose shape deviates only slightly from that of a sphere. Then, as the particle platyness increases, more and more particles align their faces along the privileged direction. This ordering phenomenon has previously been observed in experiments [35] and numerical simulations with two-dimensional elongated particles [27-29]. However, there is a difference between our findings, which confirm in a more systematic way those reported in Refs. [28,35], and those reported in Refs. [27,29], regarding the privileged direction along which the particles faces align. In our simulations, as well as in Refs. [28,35], as platyness increases, this privileged direction gradually changes from that of the major principal stress to a direction that is almost perpendicular to the shear direction, i.e., from $\theta_{m}=135^{\circ}$ to $90^{\circ}$ in Fig. 11 . In other words, as platyness increases, the larger axis of the particles tend to align with the average velocity field. In contrast, in the simulations reported in Ref. [29], the privileged direction remains that of the major principal stress, regardless of the value of the shape parameter. The difference between the observed behaviors may be attributed to the different symmetries of the tests, a simple shear device in our simulations, as well as in Refs. [28,35], and a biaxial device in those reported in Ref. [29]).

With respect to our observations, it can be hypothesized that this ordering phenomenon results from the combination of two mechanisms. On one hand, as shown in Fig. 9, the particles tend to align along the direction of the major principal stress (i.e., $\theta_{m}$ tends to $135^{\circ}$ in Fig. 11). This is convenient, since the larger forces tend to be captured by the face-face contacts, which are very stable. However, this also implies that the particles will have to rotate as the material is sheared, which is expensive in terms of energy because it implies more dilatation of the sample. On the other hand, as it has been observed in experiments with very elongated particles such as clay particles [2], the particles tend to align along a direction that is perpendicular to the shear direction (i.e., $\theta_{m}$ tends to $90^{\circ}$ in Fig. 11). This is convenient, since it does not require particle rotation. However, this implies that particles must slide with respect each other, which is expensive in terms of energy dissipation by friction. Privileged orientations that are intermediate between that of the major principal stress and that perpendicular to the shear direction (i.e., $\theta_{m}$ lying between $135^{\circ}$ and $90^{\circ}$ in Fig. 11) can thus be a consequence of a trade-off between these two mechanisms, accommodating both energy dissipation and dilation. The fact that the privileged orientation increases with the particle platyness is then explained since, as platyness increases, the first mechanism is more restrictive than the second.

We also studied the connectivity of the interaction networks in terms of the coordination number and the proportion of rattlers, i.e., particles that do not participate in the forcecarrying backbone. We found that, as the particle platyness increases, the coordination also increases and the proportion of rattlers decreases. This shows that the materials composed of the platiest particles have more connected interaction networks, even if these materials are also the loosest ones.

As is customary in this kind of investigation, we also studied the evolution of the fabric of the interaction network as well as the distribution of interaction forces. It was shown that all descriptors of the microstructure are strongly determined by the ordering phenomenon described above. Punctually, the anisotropies of interaction orientations, normal and tangential branch lengths, and normal and tangential interaction forces increase with platyness. Additionally, the principal directions of these distributions evolve in a way that is almost identical to that of the principal direction of the distribution of particle orientations. This shows that platyness, especially through this ordering phenomenon, enhances the 
ability of the system to generate anisotropic structures. A direct consequence of this anisotropic structure is the large shear strengths measured in the materials composed of very platy particles. In fact, it was shown that the shear strength of the material is well approximated by the half sum of these anisotropies and that those structural characteristics that mostly contribute to build up a large shear strength are the fabric of the interactions network and the mobilization of friction forces in these interactions. The relationship between a complex particle shape and friction mobilization has also been observed in simulations with two-dimensional angular [23-25,27], elongated [29], and nonconvex [31] particles, as well as in three-dimensional simulations [32].

As mentioned in Sec. I, this is the first paper of an ongoing investigation devoted to the mechanical behavior of clayey soils studied by DEM simulations. Specifically, the aim of this work was to explore the effect of the particle shape on the mechanical behavior and microstructure of those materials. Our perspectives are to follow on this lead while gradually enriching our model in order to make it more realistic and more comparable to clayey soils. In particular, our results with realistic interactions between clayey particles including a pore fluid will be addressed in a future publication.

\section{APPENDIX: ROTATION MOTION INTEGRATION}

Consider a particle that moves during a time step $\Delta t$. In order to integrate the particle's rotations in this time step by means of a Leapfrog algorithm, the angular velocities $\boldsymbol{\Omega}$ during the time interval $[t, t+\Delta t / 2]$ must be computed. Fincham [36] showed that this can be done using Euler equations:

$$
\boldsymbol{\Omega}_{t+\Delta t / 2}=\boldsymbol{\Omega}_{t-\Delta t / 2}+\Delta t \dot{\boldsymbol{\Omega}}_{t},
$$

where denotes time derivative. The angular accelerations are calculated as

$$
\begin{aligned}
& \dot{\boldsymbol{\Omega}}_{t}^{\alpha}=\frac{1}{J^{\alpha}}\left[\boldsymbol{\tau}_{t}^{\alpha}+\left(J^{\beta}-J^{\gamma}\right) \boldsymbol{\Omega}_{t}^{\beta} \boldsymbol{\Omega}_{t}^{\gamma}\right], \\
& \dot{\boldsymbol{\Omega}}_{t}^{\beta}=\frac{1}{J^{\beta}}\left[\boldsymbol{\tau}_{t}^{\beta}+\left(J^{\gamma}-J^{\alpha}\right) \boldsymbol{\Omega}_{t}^{\gamma} \boldsymbol{\Omega}_{t}^{\alpha}\right], \\
& \dot{\boldsymbol{\Omega}}_{t}^{\gamma}=\frac{1}{J^{\gamma}}\left[\boldsymbol{\tau}_{t}^{\gamma}+\left(J^{\alpha}-J^{\beta}\right) \boldsymbol{\Omega}_{t}^{\alpha} \boldsymbol{\Omega}_{t}^{\beta}\right],
\end{aligned}
$$

where the $J$ are particle moments of inertia and $\tau$ are the torques along particle's principal axes $\alpha, \beta$, and $\gamma$. Based on Omelyan's work [37], we developed an algorithm well suited for calculating the particle rotations using quaternions. In this algorithm, the evolution of the quaternion $\mathrm{q}$ in time is expressed as a combination of successive rotations:

$$
\mathrm{q}_{t+\Delta t}=\Delta \mathrm{q} \otimes \mathrm{q}_{t},
$$

where

$$
\Delta q^{0}=\frac{1-\Gamma}{1+\Gamma}, \quad \Delta q^{j}=\frac{\Delta t}{2(1+\Gamma)} \mathbf{\Omega}_{t+\Delta t / 2}^{j},
$$

where $j$ represents the quaternion components 1,2 , and 3 , and

$$
\Gamma=\frac{\Delta t^{2}}{16}\left[\boldsymbol{\Omega}_{t+\Delta t / 2} \cdot \boldsymbol{\Omega}_{t+\Delta t / 2}\right] .
$$

Note that this operation involves quaternion multiplications that intrinsically satisfy the normalization condition.
[1] M. D. Bolton, in Proc. International Workshop on Soil Crushability, edited by N. Y. Hyodo M. (Yamaguchi University, Japan, 1999), pp. 1-24.

[2] J. K. Mitchell and K. Soga, Fundamentals of Soil Behavior, 3rd ed. (Wiley, New York, 2005).

[3] A. Anandarajah, Eng. Geol. 47, 313 (1997).

[4] A. Anandarajah, Powder Technol. 106, 132 (1999).

[5] A. Anandarajah, Comput. Geotech. 27, 1 (2000).

[6] M. Yao and A. Anandarajah, J. Eng. Mech. 129, 585 (2003).

[7] L. Pournin, M. Weber, M. Tsukahara, J.-A. Ferrez, M. Ramaioli, and T. M. Liebling, Granular Matter 7, 119 (2005).

[8] S. A. Galindo-Torres, F. Alonso-Marroquín, Y. C. Wang, D. Pedroso, and J. D. Muñoz Castaño, Phys. Rev. E 79, 060301 (2009).

[9] F. Alonso-Marroquín, Europhys. Lett. 83, 14001 (2008).

[10] P. A. Cundall and O. D. L. Strack, Géotechnique 29, 47 (1979).

[11] P. A. Langston, M. A. Al-Awamleh, F. Y. Fraige, and B. N. Asmar, Chem. Eng. Sci. 59, 425 (2004).

[12] F. Alonso-Marroquín and Y. Wang, Granular Matter 11, 317 (2009).

[13] S. A. Galindo-Torres, D. Pedroso, D. J. Williams, and L. Li, Comput. Phys. Commun. 183, 266 (2012).

[14] J. Ghaboussi and R. Barbosa, Int. J. Num. Anal. Methods Geomech. 14, 451 (1990).
[15] F. Radjaï, F. Dubois, and F. Dubois, Discrete-Element Modeling of Granular Materials (John Wiley \& Sons, New York, 2011).

[16] GDR MiDi, Eur. Phys. J. E 14, 341 (2004).

[17] S. Luding, Granular Matter 10, 235 (2008).

[18] F. Radjai and V. Richefeu, Phil. Trans. R. Soc. A 367, 5123 (2009).

[19] K. Mair, J. Geophys. Res. 107, 2219 (2002).

[20] J. L. Anthony and C. Marone, J. Geophys. Res. 110, B08409 (2005).

[21] G. Cho, J. Dodds, and J. Santamarina, J. Geotech. Geoenviron. Eng. 132, 591 (2006).

[22] A. A. Mirghasemi, L. Rothenburg, and E. L. Matyas, Geotéchnique 52, 209 (2002).

[23] Y. Guo and J. K. Morgan, J. Geophys. Res. 109, B12305 (2004).

[24] E. Azéma, F. Radjaï, R. Peyroux, and G. Saussine, Phys. Rev. E 76, 011301 (2007).

[25] N. Estrada, E. Azéma, F. Radjai, and A. Taboada, Phys. Rev. E 84, 011306 (2011).

[26] B. Saint-Cyr, K. Szarf, C. Voivret, E. Azéma, V. Richefeu, J.-Y. Delenne, G. Combe, C. Nouguier-Lehon, P. Villard, P. Sornay, M. Chaze, and F. Radjaï, Europhys. Lett. 98, 44008 (2012).

[27] E. Azéma, N. Estrada, and F. Radjai, Phys. Rev. E 86, 041301 (2012).

[28] A. A. Peña, R. García-Rojo, and H. J. Herrmann, Granular Matter 9, 279 (2007)

[29] E. Azéma and F. Radjaï, Phys. Rev. E 81, 051304 (2010). 
[30] K. Szarf, G. Combe, and P. Villard, Powder Technol. 208, 279 (2011).

[31] B. Saint-Cyr, J.-Y. Delenne, C. Voivret, F. Radjai, and P. Sornay, Phys. Rev. E 84, 041302 (2011).

[32] E. Azéma, F. Radjai, and G. Saussine, Mech. Mater. 41, 729 (2009).

[33] P. W. Cleary, Powder Technol. 179, 144 (2008).
[34] A. Donev, F. H. Stillinger, P. M. Chaikin, and S. Torquato, Phys. Rev. Lett. 92, 255506 (2004).

[35] T. Börzsönyi, B. Szabó, G. Törös, S. Wegner, J. Török, E. Somfai, T. Bien, and R. Stannarius, Phys. Rev. Lett. 108, 228302 (2012).

[36] D. Fincham, Mol. Simul. 11, 79 (1993).

[37] I. P. Omelyan, Mol. Simul. 22, 213 (1999). 